\title{
Sintomas depressivos e fatores associados em estudantes universitários do sul do Brasil
}

\author{
Depressive symptoms and associated factors in university students of the south of Brazil
}

Rafaela Santi Dell'Osbela, Patrícia Aguiar dos Santosa, Giovana Menegata ${ }^{a}$ Fernanda Fernandes da Silva ${ }^{a}$, Paula Priscila Potter Tonietto ${ }^{a}$, Maria Luisa de Oliveira Gregoletto ${ }^{\mathrm{b}}$, Cleber Cremonese ${ }^{\mathrm{c}}$

aAcadêmica em Nutrição pelo Centro Universitário da Serra Gaúcha (FSG).

butricionista. Mestre em Saúde Coletiva. Docente do curso de Nutrição da FSG.

'Biólogo. Doutor em Ciência - Saúde Pública e Meio Ambiente. Docente do curso de Nutrição da FSG.

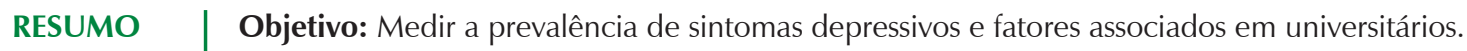

Materiais e Métodos: Realizou-se um estudo transversal, com 892 universitários de uma instituição privada do sul do Brasil. Os dados foram coletados por meio de questionário autoaplicáveis, sendo o desfecho, sintomas depressivos. Realizou-se a análise descritiva e bruta, sendo calculadas razões de prevalência (RP) com respectivos intervalos de confiança (IC) de 95\%. A análise ajustada ocorreu por regressão de Poisson, onde o modelo final foi construído a partir das variáveis com $p \leq 0,20$ na análise bruta. Análises estatísticas foram rodadas nos programas SPSS Data 23.0 e Stata 11.0.

Resultados: A média de idade foi 24,5 anos $( \pm 6,3)$, predominante o sexo feminino $(63,2 \%)$. Quanto ao desfecho, de modo geral, 30,8\% apresentaram sintomas depressivos. A análise final apresentou associação entre o desfecho e sexo feminino (RP 1,39; IC 95\% 1,09-1,76; $p=0,006)$, ser fumante ou ex-fumante (RP 1,21; IC 95\% 1,06-1,38; $p=0,004)$, realizar dietas para perda de peso 5 vezes ou mais durante o último ano (RP 1,53; IC 95\% 1,18-1,99; $p=0,003)$, insatisfação corporal (RP 1,67; IC 95\% 1,21-2,30; $p=0,002)$ e autoestima insatisfatória (RP 2,61; IC 95\% $2,14-3,18 ; p<0,001)$.

Conclusão: A alta prevalência geral de sintomas depressivos (30,8\%) identificada na população de acadêmicos encontra-se semelhante aos achados em estudo nacionais e internacionais. Aponta-se, como principais fatores associados ao desfecho, sexo feminino, fumante/ex-fumante, realizar dietas para perda de peso maior do que 5 vezes no último ano, apresentar insatisfação corporal e autoestima insatisfatória.

Palavras-chave: depressão; saúde mental; estudantes; estudos transversais; prevalência.

ABSTRACT

Objective: To measure the prevalence of depressive symptoms and associated factors in university students.

Materials and Methods: A cross-sectional study was carried out with 892 university students from a private institution in southern Brazil. The data were collected through a self-administered questionnaire, with the outcome being depressive symptoms. The descriptive and crude analysis was performed, and prevalence ratios (PR) were calculated with respective 95\% confidence intervals $(\mathrm{Cl})$. The adjusted analysis was performed by Poisson regression, where the final model was constructed from the variables with $p$-value $\leq 0.20$ in the crude analysis. Statistical analyzes were run on SPSS Data 23.0 and Stata 11.0 softwares.

Results: The mean age was 24.5 years ( \pm 6.3 ), predominantly female sex (63.2\%). Regarding the outcome, in general, $30.8 \%$ presented depressive symptoms. The final analysis showed an association between the outcome and the female sex (PR 1.39, 95\% Cl 1.09-1.76, $p=0.006$ ), being smoker or ex-smoker (PR 1.21; 95\% Cl 1.06-1.38; $p=0.004)$, diets for weight loss 5 times or more during the last year (PR 1.53, 95\% Cl 1.18-1.99, $p=0.003)$, body dissatisfaction (PR 1.67, 95\% Cl 1.21-2.30, $p=0.002)$ and unsatisfactory self-esteem (PR 2.61, 95\% Cl 2.14-3.18, $p<0.001$ ).

Conclusion: The general high prevalence of depressive symptoms (30.8\%) identified in the academic population is similar to those found in national and international studies. The main factors associated with the outcome are being female, smoker/ex-smoker, diets for weight loss more than 5 times in the last year, body dissatisfaction and unsatisfactory self-esteem.

Keywords: depression; mental health; students; cross-sectional studies; prevalence. 


\section{INTRODUÇÃO}

Os sintomas depressivos são comportamentos que antecedem e caracterizam um transtorno de humor chamado depressão ${ }^{1}$. Esses sintomas podem se manifestar através de pensamentos negativos, esgotamento, nervosismo, incapacidade de planejamentos futuros, impedimentos na concentração e sentimentos de inferioridade ${ }^{1}$. A sua presença, além de levar a depressão, pode ocasionar agravos na qualidade de vida não somente da pessoa afetada, mas também em seus familiares ${ }^{2}$.

A prevalência de depressão varia de 3 a $11 \%$ entre a população geral, e pode chegar a 34,5\% em estudantes universitários, com ênfase para o sexo feminino, onde, favorecem o surgimento desses sentimentos, aspectos de vida acadêmica, condições ambientais, psíquicas, genéticas, biológicas, além de interpessoais e institucionais ${ }^{3-5}$.

Adultos jovens, em uma instituição de ensino superior, já foram identificados com uma carga para o desenvolvimento de doenças mentais maior do que jovens da mesma faixa etária que não estão matriculados em universidades ${ }^{6}$. Adentrar no ensino superior é notoriamente uma fase de grandes mudanças e, consequentemente, de novas responsabilidades, já que a parte dos estudantes passam a ser os únicos responsáveis por administrar seu tempo, assim como muitas vezes, sua moradia e finanças ${ }^{7}$.

Além disso, tem-se uma preocupação muito grande em relação a depressão e outras doenças em adolescentes e adultos jovens, pois os agravos de saúde desenvolvidos nesta etapa da vida, tem propensão muito maior de resistência ao longo da vida ${ }^{8}$. Ainda, dentre os danos que podem perdurar na vida adulta, decorrentes da depressão, destacam-se o risco aumentado de suicídio e lesão no controle psicossocial ${ }^{9}$.

Diante desta contextualização, o objetivo do presente estudo é medir a prevalência de sintomas depressivos, bem como os fatores associados em universitários de uma instituição privada da cidade de Caxias do Sul/RS.

\section{MATERIAIS E MÉTODOS}

Estudo epidemiológico observacional com delineamento transversal, composto por uma amostra de universitários de um Centro Universitário privado da cidade de Caxias do Sul/ RS. A amostra foi determinada estimando uma prevalência de 50\% do desfecho, nível de confiança de 95\%, erro amostral de 5\%, efeito de delineamento de 1,5 e 20\% para eventuais perdas e recusas. Assim, a amostragem mínima foi calculada em 690 discentes.

Na sequência, realizou-se sorteio amostral aleatório, por conglomerado, representativo, respeitando-se a proporção de acadêmicos em cada curso de graduação da Instituição no semestre em que foram realizadas as coletas. Cursos com menos de 50 alunos foram excluídos do sorteio. Primeiramente calculou-se o número de acadêmicos necessários em cada curso. Após, estimou-se o número de turmas mínimas por curso, considerando a média de 25 acadêmicos por turma. Assim, participaram do estudo os cursos da área da saúde: biomedicina, enfermagem, educação física, fisioterapia, nutrição, odontologia, psicologia e terapia ocupacional, e de outras áreas: administração, arquitetura ciências contábeis, design, direito, engenharia ambiental, engenharia mecânica, engenharia da produção e engenharia civil. O sorteio das turmas em cada curso ocorreu de maneira aleatória. Foram incluídos no estudo universitários matriculados nas turmas sorteadas, presentes no dia da pesquisa e maiores de 18 anos. Acadêmicos com alguma incapacidade física ou cognitiva, que impedisse o preenchimento do instrumento, assim como gestantes, foram excluídos do estudo. Dessa forma, a amostra final do estudo foi constituída por 26 turmas, totalizando 892 discentes matriculados no Centro Universitário e presentes no dia da coleta de dados.

A coleta de dados ocorreu em único momento, no segundo semestre de 2016. A mesma foi realizada por uma equipe devidamente treinada, composta por quatro pessoas, estes convidaram os universitários a participar do estudo. Para a coleta de dados elaborou-se um questionário padronizado, pré-codificado, pré-testado e autoaplicável, com variáveis elaboradas pela equipe de pesquisa e com instrumentos validados. Este foi composto por questões sociodemográficas, socioeconômicas, educacionais, de estilo de vida, comportamentais, alimentares e antropométricas.

Em relação aos aspectos sociodemográficos, as seguintes variáveis foram investigadas e categorizadas: sexo (masculino; feminino), idade em anos completos (coletada de forma contínua e categorizada em quartis: $\leq 20$; 21 a 23; 24 a 26 ; $\geq 27$ ), cor da pele autodeclarada (branca; não branca) e estado civil (com companheiro; sem companheiro). Já a variável socioeconômica investigada foi: ocupação atual (com carteira assinada; outras situações). Referente ao estilo de vida, as variáveis a seguir: hábito de fumar (nunca fumou; fumante/ex-fumante) e frequência do consumo de álcool (nunca; as vezes; raramente; frequentemente). Ainda, referente a categoria educacional, duas variáveis foram investigadas: curso de graduação (coletada de forma contínua e categorizada em dicotômica: área da saúde; outras áreas) e etapa do curso (primeiro terço; segundo terço; terceiro terço).

Já as informações sobre comportamento alimentar, foram investigadas e categorizadas as seguintes variáveis: em relação ao peso atualmente está tentando (manter peso; ganhar peso; perder peso), frequência de realização de dieta 
durante o último ano (nunca; de 1 a 4 vezes; $\geq 5$ vezes), uso de remédios para emagrecer (nunca; $<1$ vez/semana, 1 vez/ semana; $\geq 2$ vezes/semana), e pular refeições (nunca; $<1$ vez/semana; 1 vez/semana; $\geq 2$ vezes/semana).

Ainda, foram investigadas medidas antropométricas de peso e altura, que foram autorreferidas, e após, calculouse o Índice de Massa Corporal (IMC), que seu cálculo se dá através da razão entre peso em quilogramas e a altura em metros ao quadrado (IMC=peso em kg/altura em $\mathrm{m}^{2}$ ), e que é classificado com base na Organização Mundial da Saúde (OMS). Fazendo uso dessa referência, as informações foram coletadas de forma contínua e categorizada conforme a classificação, sem excesso de peso $\left(\leq 24,99 \mathrm{~kg} / \mathrm{m}^{2}\right)$ e com excesso de peso $\left(\geq 25 \mathrm{~kg} / \mathrm{m}^{2}\right)^{10}$. Referente a sentimentos, a variável insatisfação corporal (não; sim) foi investigada e categorizada. Assim como a variável autoestima (satisfatória; insatisfatória), sendo que a mesma foi avaliada conforme a "Escala de Autoestima de Rosenberg" (EAR) ${ }^{11}$. Em virtude da análise estatística, utilizou-se a mediana da EAR para classificar os universitários com alta (satisfatória) e baixa autoestima (insatisfatória). Dessa forma, os estudantes com escore $\geq 26$ foram inseridos na classificação de autoestima satisfatória, e $\leq 25$ pontos, classificadas como insatisfatória ${ }^{12}$.

Para a avaliação da variável desfecho, sintomas depressivos, utilizou-se o instrumento validado por Major Depression Inventory (MDI), traduzido e adaptado para a língua portuguesa por Parcias et al. ${ }^{4}$ Este instrumento abrange 10 sintomas de depressão, conforme a $10^{\text {a }}$ Classificação Internacional de Doenças (CID-10), o qual tem o papel de fazer as classificações da Organização Mundial da Saúde (OMS) - CID e Classificação Internacional de Funcionalidade, Incapacidade e Saúde (CIF) nas definições de deficiência e incapacidade ${ }^{13}$, assim como, avalia a severidade dos sintomas depressivos. Os itens 8 e 10 do MDI apresentam subitens "a" e "b", e somente é considerada a maior pontuação entre eles. A avaliação é realizada pela pontuação final, onde escores com maior pontuação, identificam maior presença dos sintomas depressivos. A variância da pontuação atinge entre 0 e 50, sendo que escores acima 16 foram considerados como risco de depressão $(\mathrm{MDI}+)^{4}$. Assim, foi investigada a variável sintomas depressivos (não apresenta; apresenta).

A construção do banco de dados e as análises estatísticas foram realizadas por meio dos programas SPSS Statistic Data 23.0 (Statistical Package for Social Sciences - Chicago, IL, 2008) e Stata 11. A análise descritiva foi realizada por meio de valores absolutos, frequências e prevalências do desfecho. $\mathrm{Na}$ análise bruta calculou-se as razões de prevalência (RP) e respectivos intervalos de confiança (IC) de 95\%, com um nível de significância de $5 \%(p \leq 0,05)$ para identificar associação entre o desfecho e as variáveis de exposição.
Posteriormente, realizou-se a análise ajustada por regressão de Poisson, utilizando-se a técnica de backwards. Assim, as variáveis com até $20 \%$ de significância $(p \leq 0,20)$, na análise bruta, foram incluídas no modelo final.

O projeto de pesquisa foi aprovado por um Comitê de Ética em Pesquisa, de acordo com a Resolução no 466/12 do Conselho Nacional de Saúde, por meio do CAAE 56285316.5.0000.5668, sob no do parecer 1.589.809. Todos os acadêmicos participantes do estudo leram e assinaram um termo de consentimento livre e esclarecido (TCLE), o qual autorizou a participação e publicação dos resultados em anonimato.

\section{RESULTADOS}

A amostra foi composta por 892 discentes, o que corresponde a $12,7 \%$ dos universitários matriculados na instituição de ensino durante o período da coleta de dados (2016).

Entre os estudantes houve um predomínio do sexo feminino $(63,2 \% ; n=564)$ e com a cor de pele branca $(85,5 \% ; n=757)$. A idade média foi de 24,5 (DP $\pm 6,3)$ anos e a prevalência foi de acadêmicos com idade $\geq 27$ anos $(29,4 \% ; n=262)$. Já na variável ocupação, 51,8\% $(n=462)$ responderam estar com carteira assinada. Sobre as variáveis de estilo de vida, 89,3\% ( $n=792)$ nunca fumou, e 48,4\% $(n=394)$ relataram nunca ou raramente consumir bebidas alcoólicas. E quanto ao curso, 41,4\% $(n=369)$ eram da área da saúde, e 44,4\% (n=394) externou estar no segundo terço, em relação a etapa que se encontrava (Tabela 1).

No que se refere as variáveis para comportamento alimentar, 43,9\% $(n=389)$ disseram que estavam tentando perder peso, mas 53,9\% $(n=481)$ disse não ter realizado dieta para perda de peso no último ano. Em relação ao uso de medicamentos e pular refeições com o objetivo de emagrecimento, 95,1\% $(n=842)$ e $77,7 \%(n=688)$, respectivamente, relataram nunca terem usado esses métodos. Na análise de IMC houve a prevalência para estudantes sem excesso de peso, representando 69,1\% $(n=598)$ da amostra. A insatisfação corporal foi relatada em $75,3 \%(n=654)$ dos acadêmicos e apenas 5,6\% $(n=48)$ apresentaram autoestima insatisfatória (Tabela 2).

Em relação ao desfecho investigado, 30,8\% ( $n=275)$ dos acadêmicos apresentaram sintomas depressivos (valor não demonstrado nas tabelas). Na análise da razão de prevalência, trazidas nas Tabelas 1 e 2, foram observadas associações significativas com as variáveis sexo, idade, hábito de fumar, qual a tentativa em relação ao peso (manter, ganhar ou perder peso), frequência de dieta no último ano, uso de medicamentos para emagrecer, frequência que costuma pular refeições, IMC, insatisfação corporal e autoestima. 
Referente ao sexo, mulheres apresentaram uma prevalência 59\% maior que os homens (RP: 1,59; IC 95\%: 1,26-2,00), já indivíduos com 27 anos ou mais expressaram proteção de $29 \%$ quando comparados aos mais jovens (RP: 0,71; IC 95\%: 0,53-0,95). Fumantes ou ex-fumantes tiveram $22 \%$ a mais do desfecho em relação aos que nunca fumaram (RP: 1,22; IC 95\%: 1,07-1,38) (Tabela 1).

Quanto ao peso, houve uma prevalência de $55 \%$ do desfecho a mais naqueles que estavam tentando perder peso do que naqueles que queriam manter ou ganhar peso (RP: 1,55; IC 95\%: 1,24-1,94). Observou-se ainda, que o aumento do número de frequências de dieta, esteve associado de forma positiva com a presença de SD, com $37 \%$ e $81 \%$ a mais de prevalência, respectivamente, nos acadêmicos com 1 a 4 e 5 ou mais vezes de dieta, em relação aqueles que nunca realizaram dietas durante o último ano (RP: 1,37; IC 95\%: 1,10-1,71) (RP: 1,81; IC 95\%: 1,39-2,34) (Tabela 2).

No que diz respeito ao uso de medicamentos para emagrecer, identificou-se relação positiva de 95\% e aproximadamente o triplo de prevalência com relação aos $\mathrm{SD}$, respectivamente, nos acadêmicos que fazem uso 2 ou

Tabela 1. Variáveis sociodemográficas, sócioeconômicas, educacionais e estilo de vida em relação à prevalência de sintomas depressivos (SD) em universitários de uma instituição particular do município de Caxias do Sul, RS (n=892).

\begin{tabular}{|c|c|c|c|c|}
\hline Variáveis de exposição & n (\%) & Prevalência de SD & RP bruta (IC 95\%) & Valor de $p^{*}$ \\
\hline Sexo & & & & 0,000 \\
\hline Masculino & $328(36,8)$ & 22,5 & 1 & \\
\hline Feminino & $564(63,2)$ & 35,7 & $1,59(1,26-2,00)$ & \\
\hline Idade/anos $(x=24,5 \pm 6,3)$ & & & & 0,008 \\
\hline$\leq 20$ & $230(25,8)$ & 31,9 & 1 & \\
\hline 21 a 23 & $256(28,7)$ & 36,6 & $1,14(0,89-1,47)$ & \\
\hline 24 a 26 & $144(16,1)$ & 33,6 & $1,05(0,77-1,42)$ & \\
\hline$\geq 27$ & $262(29,4)$ & 22,8 & $0,71(0,53-0,95)$ & \\
\hline Cor de pele & & & & 0,190 \\
\hline Branca & $757(85,5)$ & 29,8 & 1 & \\
\hline Não branca & $128(14,5)$ & 35,4 & $1,18(0,91-1,54)$ & \\
\hline Estado civil & & & & 0,421 \\
\hline Com companheiro(a) & $185(21,2)$ & 28,2 & 1 & \\
\hline Sem companheiro(a) & $689(78,8)$ & 31,3 & $1,11(0,86-1,43)$ & \\
\hline Ocupação atual & & & & 0,960 \\
\hline Com carteira assinada & $462(51,8)$ & 30,9 & 1 & \\
\hline Outras situações & $430(48,2)$ & 30,8 & $0,99(0,81-1,21)$ & \\
\hline Hábito de fumar & & & & 0,002 \\
\hline Nunca fumou & $792(89,3)$ & 29,2 & 1 & \\
\hline Fumante/ex-fumante & $95(10,7)$ & 43,6 & $1,22(1,07-1,38)$ & \\
\hline Consumo álcool & & & & 0,377 \\
\hline Nunca & $142(16,0)$ & 29,8 & 1 & \\
\hline Raramente & $288(32,4)$ & 27,6 & $0,92(0,67-1,26)$ & \\
\hline Às vezes & $394(44,3)$ & 33,0 & $1,10(0,82-1,48)$ & \\
\hline Frequentemente & $65(7,3)$ & 35,9 & $1,20(0,79-1,82)$ & \\
\hline Curso de graduação & & & & 0,314 \\
\hline Área da saúde & $369(41,4)$ & 32,7 & 1 & \\
\hline Outras áreas & $523(58,6)$ & 29,5 & $0,90(0,74-1,10)$ & \\
\hline Etapa do curso & & & & 0,316 \\
\hline Primeiro terço & $187(21,1)$ & 34,2 & 1 & \\
\hline Segundo terço & $394(44,4)$ & 31,8 & $0,92(0,72-1,18)$ & \\
\hline Terceiro terço & $307(34,6)$ & 28,0 & $0,81(0,62-1,06)$ & \\
\hline
\end{tabular}

RP: razão de prevalência.

*Teste qui-quadrado para heterogeneidade. Valores em negrito são estatisticamente significativos $(p \leq 0,05)$. 
Tabela 2. Variáveis de comportamento alimentar, estilo de vida e medidas antropométricas em relação à prevalência de sintomas depressivos (SD) em universitários de uma instituição particular do município de Caxias do Sul, RS (n=892).

\begin{tabular}{|c|c|c|c|c|}
\hline Variáveis de exposição & n (\%) & Prevalência de SD & RP bruta (IC 95\%) & Valor de $p^{*}$ \\
\hline Em relação ao peso está tentando & & & & $<0,001$ \\
\hline Manter peso & $386(43,5)$ & 23,9 & 1 & \\
\hline Ganhar peso & $112(12,6)$ & 31,5 & $1,31(0,94-1,82)$ & \\
\hline Perder peso & $389(43,9)$ & 37,3 & $1,55(1,24-1,94)$ & \\
\hline Frequência de dieta/último ano & & & & $<0,001$ \\
\hline Nunca & $481(53,9)$ & 25,1 & 1 & \\
\hline 1 a 4 vezes & $300(33,6)$ & 34,6 & $1,37(1,10-1,71)$ & \\
\hline$\geq 5$ vezes & $111(12,4)$ & 45,5 & $1,81(1,39-2,34)$ & \\
\hline Uso de medicamentos para emagrecer/último ano & & & & $<0,001$ \\
\hline Nunca & $842(95,1)$ & 29,3 & 1 & \\
\hline$<1$ vez/semana & $16(1,8)$ & 81,3 & $2,77(2,14-3,59)$ & \\
\hline $1 \mathrm{vez} / \mathrm{semana}$ & $5(0,6)$ & 40,0 & $1,36(0,46-4,02)$ & \\
\hline$\geq 2$ vezes/semana & $22(2,5)$ & 57,1 & $1,95(1,32-2,86)$ & \\
\hline Pular refeições & & & & $<0,001$ \\
\hline Nunca & $688(77,7)$ & 26,1 & 1 & \\
\hline$<1$ vez/semana & $95(10,7)$ & 47,9 & $1,83(1,43-2,34)$ & \\
\hline $1 \mathrm{vez} / \mathrm{semana}$ & $38(4,3)$ & 36,8 & $1,40(0,91-2,17)$ & \\
\hline$\geq 2$ vezes/semana & $64(7,2)$ & 52,4 & $2,00(1,53-2,61)$ & \\
\hline $\mathrm{IMC}(\mathrm{x}=23,8 \pm 3,8)$ & & & & 0,004 \\
\hline Sem excesso de peso & $598(69,1)$ & 28,1 & 1 & \\
\hline Com excesso de peso & $267(30,9)$ & 37,8 & $1,34(1,09-1,64)$ & \\
\hline Insatisfação corporal & & & & $<0,001$ \\
\hline Não & $214(24,7)$ & 17,0 & 1 & \\
\hline Sim & $654(75,3)$ & 35,6 & $2,09(1,52-2,87)$ & \\
\hline Autoestima & & & & $<0,001$ \\
\hline Satisfatória & $808(94,4)$ & 27,4 & 1 & \\
\hline Insatisfatória & $48(5,6)$ & 87,2 & $3,18(2,72-3,73)$ & \\
\hline
\end{tabular}

mais vezes por semana e naqueles que usam menos de 1 vez por semana, em comparação aqueles que nunca usaram ou usam 1 vez por semana esse tipo de medicação (RP: 1,95; IC 95\%: 1,32-2,86) (RP: 2,77; IC 95\%: 2,14-3,59) (Tabela 2).

O hábito de pular refeições quando confrontado com aqueles que nunca pularam refeições ou pularam apenas 1 vez durante a semana, se mostrou o dobro mais prevalente naqueles que o faziam 2 ou mais vezes por semana, e $83 \%$ nos que demonstraram essa prática menos de 1 vez por semana (RP: 2,00; IC 95\%: 1,53-2,61) (RP: 1,83; IC 95\%: 1,43-2,34). Quanto ao IMC, universitários com excesso de peso apresentaram uma prevalência 34\% maior em relação aos sem excesso de peso (RP: 1,34; IC 95\%: 1,09-1,64). Já em relação a insatisfação corporal, a prevalência foi o dobro maior nos discentes que apresentaram insatisfação, do que naqueles que não apresentaram (RP: 2,09; IC 95\%: 1,52-2,87). Do mesmo modo, discentes que relataram ter autoestima insatisfatória, tiveram uma prevalência aproximadamente o triplo maior, em relação aqueles que apresentaram autoestima satisfatória (RP: 3,18; IC 95\%: 2,72-3,73).

Após realizar a análise ajustada (Tabela 3) identificou-se associações significativas entre o desfecho e sexo, hábito de fumar, frequência de realização de dieta durante o último ano, insatisfação corporal e autoestima. Os discentes do sexo feminino apresentavam 39\% mais probabilidade de desenvolver o desfecho (RP: 1,39; IC 95\%: 1,09-1,76; $p=0,006)$ quando comparados aos do sexo masculino. Quanto ao hábito de fumar, observou-se $21 \%$ maior possibilidade de desenvolver sintomas depressivos em discentes fumantes ou ex-fumantes (RP: 1,21; 
Tabela 3. Análise da razão de prevalência ajustada em relação ao desfecho sintomas depressivos em universitários de uma instituição particular do município de Caxias do Sul, RS ( $n=892)$.

\begin{tabular}{|c|c|c|}
\hline Variáveis de exposição & $\begin{array}{l}\text { RP* ajustada } \\
\quad \text { (IC 95\%) }\end{array}$ & $\begin{array}{l}\text { Valor de } \\
\qquad p^{* *}\end{array}$ \\
\hline Sexo & & 0,006 \\
\hline Masculino & 1 & \\
\hline Feminino & $1,39(1,09-1,76)$ & \\
\hline Idade/anos $(x=24,5 \pm 6,3)$ & & 0,082 \\
\hline$\leq 20$ & 1 & \\
\hline 21 a 23 & $1,28(1,00-1,64)$ & \\
\hline 24 a 26 & $1,23(0,92-1,65)$ & \\
\hline$\geq 27$ & $0,80(0,59-1,09)$ & \\
\hline Cor de pele & & 0,227 \\
\hline Branca & 1 & \\
\hline Não branca & $1,18(0,90-1,56)$ & \\
\hline Hábito de fumar & & 0,004 \\
\hline Nunca fumou & 1 & \\
\hline Fumante/ex-fumante & $1,21(1,06-1,38)$ & \\
\hline Em relação ao peso está tentando & & 0,471 \\
\hline Manter peso & 1 & \\
\hline Ganhar peso & $1,28(0,91-1,80)$ & \\
\hline Perder peso & $0,89(0,70-1,15)$ & \\
\hline Frequência de dieta/último ano & & 0,003 \\
\hline Nunca & 1 & \\
\hline 1 a 4 vezes & $1,09(0,87-1,37)$ & \\
\hline$\geq 5$ vezes & $1,53(1,18-1,99)$ & \\
\hline $\begin{array}{l}\text { Uso de medicamentos para } \\
\text { emagrecer/último ano }\end{array}$ & & 0,222 \\
\hline Nunca & 1 & \\
\hline$<1 \mathrm{vez} /$ semana & $1,54(1,01-2,33)$ & \\
\hline $1 \mathrm{vez} /$ semana & $1,18(0,30-4,55)$ & \\
\hline$\geq 2$ vezes/semana & $1,03(0,67-1,58)$ & \\
\hline Pular refeições & & 0,058 \\
\hline Nunca & 1 & \\
\hline$<1$ vez/semana & $1,42(1,11-1,84)$ & \\
\hline $1 \mathrm{vez} / \mathrm{semana}$ & $1,00(0,67-1,49)$ & \\
\hline$\geq 2$ vezes/semana & $1,35(1,02-1,80)$ & \\
\hline $\operatorname{IMC}(x=23,8 \pm 3,8)$ & & 0,263 \\
\hline Sem excesso de peso & 1 & \\
\hline Com excesso de peso & $1,14(0,90-1,44)$ & \\
\hline Insatisfação corporal & & 0,002 \\
\hline Não & 1 & \\
\hline Sim & $1,67(1,21-2,30)$ & \\
\hline Autoestima & & $<0,001$ \\
\hline Satisfatória & 1 & \\
\hline Insatisfatória & $2,61(2,14-3,18)$ & \\
\hline
\end{tabular}

* RP: razão de prevalência.

**Valores em negrito são estaticamente significativos $(p \leq 0,05)$.

Modelo final ajustado pelo método de regressão de Poisson, por todas as variáveis que apresentaram valor de $p \leq 0,20$ na análise bruta.
IC 95\%: 1,06-1,38; $p=0,004)$. Em relação a frequência de realização de dieta, os discentes que realizaram 5 vezes ou mais durante o último ano apresentaram maior probabilidade de desenvolver o desfecho (53\%) (RP: 1,53; IC 95\%: 1,18-1,99; $p=0,003)$. Já os discentes com insatisfação corporal e autoestima insuficiente apresentaram aproximadamente $67 \%$ e 2,6 vezes mais possibilidade de desenvolver o desfecho, respectivamente (RP: 1,67; IC 95\%: 1,21-2,30; $p=0,002) \quad(\mathrm{RP}: 2,61$; IC 95\%: 2,14-3,18; $p<0,001)$.

\section{DISCUSSÃO}

No que se refere ao objetivo específico do estudo, em que se pretendia investigar a prevalência de sintomas depressivos e fatores associados dos universitários, os resultados apontaram significância com as variáveis sociodemográficas, referentes ao estilo de vida e comportamento alimentar. A importância de estudos sobre sintomas depressivos, se dá em consequência de que os mesmos podem causar efeitos negativos na vida de jovens adultos, como por exemplo, prejudicar o desempenho acadêmico, com possíveis consequências para toda a vida adulta ${ }^{8}$. Ainda, o contexto ao qual os universitários estão inseridos, com as elevada demanda acadêmica e altos níveis de estresse, faz com que muitos desenvolvam este tipo de sintomas ${ }^{14}$.

Em relação ao desfecho, 30,8\% dos universitários entrevistados apresentaram sintomas depressivos. Pesquisa realizada por Aragão et al. ${ }^{1}$, em universidades de Sergipe, no Brasil, com 215 estudantes de idades entre 19 e 24 anos, a qual identificou uma prevalência de $29,7 \%$ de sintomas depressivos. Contribuindo com estes achados, mas com faixa etária diferente, Khan et al. ${ }^{15}$, em pesquisa realizada em oito escolas secundárias da capital de Bangladesh, com 898 alunos de 13 a 16 anos de idade, mostrou prevalência de 25\% de presença de sintomas depressivos. Dados semelhantes foram encontrados por Jaalouk et al. ${ }^{16}$, em estudo realizado no Líbano, com 457 estudantes de graduação, de 18 a 33 anos, em que $24,1 \%$ apresentaram sintomas depressivos. Desse modo, observa-se que as prevalências identificadas se assemelham ao presente estudo ${ }^{1,15,16}$. Já em pesquisa de Sa-Junior et al. ${ }^{17}$, em 100 instituições de ensino superior, das 27 capitais brasileiras, com 12.711 universitários, de faixa etária entre 17 e 31 anos, mostrou que 79,9\% dos participantes respondeu ter pelo menos um sintoma depressivo, ou seja, expuseram sintomas depressivos leves.

Portanto, destacam-se os universitários como uma população de risco e prevalência elevada de sintomas depressivos, uma vez que estes precisam se adaptar a uma nova realidade de forma rápida, já que o ingresso no nível superior faz com que muitos precisem sair de 
suas moradias e ir para diferentes lugares, além de fazer longos deslocamentos, insucesso acadêmico e períodos sobrecarregados de trabalhos e provas, o que gera uma insatisfação e aumenta o estresse ${ }^{18}$.

Os resultados do presente estudo apontaram que a maior prevalência de sintomas depressivos foi entre o sexo feminino (39\%). Corroboram com este achado a pesquisa de Cremasco \& Baptista $^{19}$, realizada em uma universidade de Minas Gerais, com participação de 77 alunos, este evidenciou que mulheres são mais propensas a desenvolverem transtornos depressivos. Ainda, Pereira ${ }^{18}$ destaca, que mulheres são mais predispostas a esses tipos de sintomas, pois apresentam uma expectativa superior em relação ao futuro, e quando as mesmas não são alcançadas, geram desânimo e causam reações depressivas. Apoiando os achados, Khan et al. ${ }^{15}$, em seu estudo com adolescentes, já caracterizado a cima, também encontrou maior prevalência de sintomas depressivos no sexo feminino (30\%). Assim, sugere-se que estudantes do sexo feminino apresentem maiores chances de desenvolver sintomas depressivos durante o ensino superior, possivelmente devido as maiores expectativas, ocasionando desânimo e frustração.

Ainda, poucos são os estudos que investigam uma possível relação entre hábito de fumar e sintomas depressivos, já que a maioria deles faz essa relação com o consumo de álcool. Acredita-se que os SD podem influenciar de maneira significativa o hábito de fumar nos indivíduos, uma vez que os mesmos pensam que o tabagismo reduz o sofrimento psicológico ${ }^{20}$. Sobre essa perspectiva, o presente estudo encontrou uma $\mathrm{RP}=1,21$ maior de $\mathrm{SD}$ nos fumantes e exfumantes em comparação aos que nunca fumaram. Porém, em um estudo por conveniência, realizado por Saravanan \& Heidhy ${ }^{20}$, com 719 universitários da Malásia, que apresentaram uma idade média de 21,6 anos, demostrou que a depressão não teve ligação com a dependência a nicotina, mas os estudantes que fumavam, mostraram em suas respostas poder pensar que o cigarro ajuda a diminuir a depressão.

A frequência de realização de dietas no último ano, foi apontada neste estudo com 53\% a mais do desfecho, naqueles que realizaram 5 vezes ou mais essa prática, quando comparados aos que nunca a fizeram, ou realizaram de $1 \mathrm{a}$ 4 vezes durante o último ano. Um estudo de Loth et al. ${ }^{21}$, revelou que um conjunto de variáveis pode estar associadas a realização de dietas na adolescência e vida adulta, estando entre eles, a presença de sintomas depressivos, além da preocupação com o peso e importância que o peso atual tem na vida de muitas dessas pessoas. Acredita-se que a elevada frequência de realização de dietas no último ano nos permita identificar a excessiva preocupação com o peso, possivelmente facilitando o surgimento de sintomas depressivos nessa população.

Preocupar-se com o próprio corpo tornou-se uma realidade em diversos países, onde indivíduos procuram o corpo ideal, juntamente a isso, a insatisfação corporal também está muito presente, sendo esta, um sentimento negativo apresentado por ambos os sexos ${ }^{22}$. O presente estudo encontrou uma $\mathrm{RP}=1,67$ maior de sintomas depressivos nos acadêmicos que apresentaram insatisfação corporal, quando comparados aos que não apresentaram. Em um estudo realizado por Bordignon \& Teodoro ${ }^{23}$, com 79 crianças, de idade entre 7 e 12 anos, no Rio Grande do Sul, $77,2 \%$ dos entrevistados demonstrou estar insatisfeito com o corpo. Dados semelhantes foram encontrados por Marques et al. ${ }^{24}$, em pesquisa realizada com adolescentes apresentando idade entre 10 e 16 anos, na cidade de Itajaí-SC, onde a prevalência de insatisfação corporal esteve presente em $74,3 \%$ da amostra total de 214 alunos. Ambos os estudos, apesar da diferença na faixa etária, demonstraram significância estatística na comparação da insatisfação corporal e sintomas depressivos, corroborando assim, com os achados desta pesquisa. Ainda, Alvarenga et al. ${ }^{25}$, com uma amostra de 2.402 estudantes de universidades de 5 regiões do Brasil, encontrou uma prevalência de $64,4 \%$ de insatisfação corporal entre as universitárias. Desse modo, sugere-se que a insatisfação corporal e a busca por um corpo ideal possivelmente favoreçam o surgimento do desfecho em universitários.

Apesar de um número pequeno de alunos apresentarem autoestima insatisfatória nesta pesquisa $(5,6 \%)$, quando analisada em relação ao desfecho, apresentaram uma prevalência aproximadamente 2,5 vezes maior de sintomas depressivos em relação aqueles com autoestima satisfatória. Apoia essa hipótese, o estudo realizado por Furegato et al. ${ }^{26}$, com 224 acadêmicos do curso de enfermagem da universidade de Ribeirão Preto em São Paulo, onde, dos 19,2\% dos estudantes que apresentaram baixa e média autoestima, ou seja, autoestima insatisfatória, todos apresentavam sintomas depressivos. Portanto, acredita-se que a autoestima insatisfatória seja possivelmente um fator de risco o surgimento de sintomas depressivos na população estudada.

Quanto as limitações deste estudo, salienta-se o delineamento transversal, que impossibilita o acompanhamento dos indivíduos, uma vez que os sentimentos descritos são os momentâneos, podendo assim resultar em causalidade reversa, podendo um estudo longitudinal reduzir essa possibilidade. O viés da memória, assim como do tempo levado para responder o instrumento de coleta e possíveis omissões, por ser realizado em sala de aula, com demais 
colegas próximos, é outro aspecto de possível limitação. Ainda, a inexistência de alguma variável controle, exposição ou confundimento, devem ser julgados como limitantes de investigações.

Destaca-se, positivamente, a dimensão amostral, a qual representou aproximadamente $13 \%$ dos acadêmicos do Centro Universitário onde o estudo foi realizado, atingindo assim um percentual muito representativo da realidade institucional. Além disso, acredita-se tratar de um dos primeiros estudos com esta população na região Sul, que avalie os sintomas depressivos em ambos os sexos e de cursos distintos, sendo possível assim um melhor entendimento no assunto e o desenvolvimento de ações de prevenção e discussão para futuras pesquisas.

Portanto, identificou-se uma alta prevalência de sintomas depressivos na população de acadêmicos, resultado semelhante aos achados em estudos nacionais e internacionais com populações semelhantes. Ainda, o estudo apontou como principais determinantes, associados ao aparecimento do desfecho, ser do sexo feminino, fumante/ ex-fumante, realizar dietas para perda de peso $\geq 5$ vezes no último ano, insatisfação corporal e autoestima insatisfatória. Assim, reitera-se a importância de investigar populações semelhantes a aqui apresentada, em estudos transversais e longitudinais, a fim de fortalecer possíveis achado do presente estudo, na perspectiva de realizar ações preventivas e/ou de acompanhamento daqueles indivíduos mais vulneráveis. Por fim, o relatório geral da pesquisa foi entregue ao coordenador de ensino da instituição envolvida no estudo, com sugestões de ações junto aos acadêmicos investigados.

\section{REFERÊNCIAS}

1. Aragão JA, Freire MRM, Farias LGN, Diniz SS, Aragão FMS, Aragão ICS, Lima TB, Reis FP. Prevalence of depressive symptoms among medical students taught using problem-based learning versus traditional methods. Int J Psychiatry Clin Pract. 2018;22(2):123-8. https://doi.org/10.1080/13651501.2017.1383438

2. Grolli V, Wagner MF, Dalbosco SNP. Sintomas depressivos e de ansiedade em adolescentes do ensino médio. Rev Psicol IMED. 2017;9(1):87-103. https://doi.org/10.18256/2175-5027.2017. v9i1.2123

3. Acharya L, Jin L, Collins W. College life is stressful today - Emerging stressors and depressive symptoms in college students. J Am Coll Health. 2018 Mar 22:1-10. https://doi.org/10.1080/07448481.20 18.1451869

4. Parcias S, Rosario BPd, Sakae T, Monte F, Guimarães AC, Xavier AJ. Validação da versão em português do Inventário de Depressão Maior. J Bras Psiquiatr. 2011;60(3):164-70. https://doi.org/10.1590/ S0047-20852011000300003

5. Horgan A, Kelly P, Goodwin J, Behan L. Depressive Symptoms and Suicidal Ideation among Irish Undergraduate College Students. Issues Ment Health Nurs. 2018;39(7):575-84. https://doi.org/10.10 80/01612840.2017.1422199
6. Piumatti G. Motivation, health-related lifestyles and depression among university students: A longitudinal analysis. Psychiatry Res. 2018;260:412-17. https://doi.org/10.1016/j.psychres.2017. 12.009

7. Perez PM, De Castro IR, Franco AS, Bandoni DH, Wolkoff DB. Dietary practices of quota and non-quota students at a Brazilian public university. Ciênc Saúde Colet. 2016;21(2):531-42. https:// doi.org/10.1590/1413-81232015212.01732015

8. Shi M, Liu L, Wang ZY, Wang L. Prevalence of depressive symptoms and its correlations with positive psychological variables among Chinese medical students: an exploratory cross: sectional study. BMC Psychiatry. 2016;16:3. https://doi.org/10.1186/s12888-0160710-3

9. Larsson B, Ingul J, Jozefiak T, Leikanger E, Sund AM. Prevalence, stability, 1-year incidence and predictors of depressive symptoms among Norwegian adolescents in the general population as measured by the Short Mood and Feelings Questionnaire. Nord J Psychiatry. 2016;70(4):290-6. https://doi.org/10.3109/08039488.2 015.1109137

10. Ministério da Saúde (BR). Secretaria de Atenção à Saúde. Departamento de Atenção Básica. Orientações para a coleta e análise de dados antropométricos em serviços de saúde: norma técnica do Sistema de Vigilância Alimentar e Nutricional-SISVAN [Internet]. Brasília: Ministério da Saúde; 2011 [Capturado 2018 mar 07]. Disponível em: http://bvsms.saude.gov.br/bvs/publicacoes/ orientacoes_coleta_analise_dados_antropometricos.pdf.

11. Sbicigo JB, Bandeira DR, Dell'Aglio DD. Escala de Autoestima de Rosenberg (EAR): validade fatorial e consistência interna. Psico-USF. 2010;15(3):395-403. https://doi.org/10.1590/S141382712010000300012

12. Fortes LDS, Cipriani FM, Coelho FD, Paes ST, Ferreira MEC. A autoestima afeta a insatisfação corporal em adolescentes do sexo feminino? Rev Paul Pediatr. 2014;32(3):236-340. https://doi. org/10.1590/0103-0582201432314

13. Nubila D, Ventura HB, Buchalla CM. O papel das Classificações da OMS-CID e CIF nas definições de deficiência e incapacidade. Rev Bras Epidemiol. 2008;11(2):324-35. https://doi.org/10.1590/ S1415-790X2008000200014

14. Barker ET, Howard AL, Villemaire-Krajden R, Galambos NL. The Rise and Fall of Depressive Symptoms and Academic Stress in Two Samples of University Students. J Youth Adolesc. 2018;47(6): 1252-66. https://doi.org/10.1007/s10964-018-0822-9

15. Khan A, Ahmed R, Burton NW. Prevalence and correlates of depressive symptoms in secondary school children in Dhaka city, Bangladesh. Ethn Health. 2017;1-13. https://doi.org/10.1080/135 57858.2017.1398313

16. Jaalouk D, Matar Boumosleh J, Helou L, Abou Jaoude M. Dietary patterns, their covariates, and associations with severity of depressive symptoms among university students in Lebanon: a crosssectional study. Eur J Nutr. 2018. https://doi.org/10.1007/s00394018-1614-4

17. Sa-Junior ARd, de Andrade AG, Andrade LH, Gorenstein C, Wang YP. Response pattern of depressive symptoms among college students: What lies behind items of the Beck Depression InventoryII? J Affect Disord. 2018;234:124-30. https://doi.org/10.1016/j. jad.2018.02.064 
18. Pereira AAM. Dor psicológica e ideação suicida em estudantes [dissertação] [Internet]. Aveiro: Universidade de Aveiro; 2013. [Capturado 2018 maio 14]; Disponível em: https://ria.ua.pt/ handle/10773/11527

19. Cremasco GdS, Baptista MN. Depressão, motivos para viver e o significado do suicídio em graduandos do curso de psicologia. Estud Interdiscip Psicol. 2017;8(1):22-37. https://doi.org/10.5433/22366407.2017v8n1p22

20. Saravanan C, Heidhy I. Psychological problems and psychosocial predictors of cigarette smoking behavior among undergraduate students in Malaysia. Asian Pac J Cancer Prev. 2014;15(18): 7629-34. https://doi.org/10.7314/APJCP.2014.15.18.7629

21. Loth KA, MacLehose R, Bucchianeri M, Crow S, Neumark-Sztainer D. Predictors of dieting and disordered eating behaviors from adolescence to young adulthood. J Adolesc Health. 2014;55(5): 705-12. https://doi.org/10.1016/j.jadohealth.2014.04.016

22. Carvalho PHBd, Oliveira FdC, Neves CM, Meireles JFF, Lopes VGV, Ferreira MEC. Busca pela "muscularidade" e variáveis associadas em adultos jovens. Rev Bras Ciênc Esporte. 2016;22(2):118-21. https://doi.org/10.1590/1517-869220162202142544

23. Bordignon S, Teodoro MLM. Relações entre percepção corporal, autoconceito e traços depressivos em crianças escolares com e sem excesso de peso. Aletheia. 2011;(34):19-31.

24. Marques FDA, Legal EJ, Höfelmann DA. Insatisfação corporal e transtornos mentais comuns em adolescentes. Rev Paul Pediatr. 2012;30(4):553-61. https://doi.org/10.1590/S0103-05822012 000400014

25. Alvarenga MdS, Philippi ST, Lourenço BH, Sato PdM, Scagliusi FB. Insatisfação com a imagem corporal em universitárias brasileiras. J Bras Psiquiatr. 2010;59(1):44-51. https://doi.org/10.1590/S004720852010000100007

26. Furegato ARF, Silva ECd, Campos MCd, Cassiano RPdT. Depressão e auto-estima entre acadêmicos de enfermagem. Rev Psiquiatr Clín. (São Paulo). 2006;33(5):239-44. https://doi.org/10.1590/S010160832006000500003 